\title{
Communicating without a functioning language system: Implications for the role of language in mentalizing
}

\author{
Roel M. Willems ${ }^{\mathrm{a}, *}$, Yael Benn $^{\mathrm{b}}$, Peter Hagoort ${ }^{\mathrm{a}, \mathrm{c}}$, Ivan Toni ${ }^{\mathrm{a}}$, Rosemary Varley ${ }^{\mathrm{b}}$ \\ a Donders Institute for Brain, Cognition and Behaviour, Radboud University Nijmegen, P.O. Box 9101, 6500 HB Nijmegen, The Netherlands \\ ${ }^{\mathrm{b}}$ Human Communication Sciences, University of Sheffield, UK \\ c Max Planck Institute for Psycholinguistics, Nijmegen, The Netherlands
}

\section{A R T I C L E I N F O}

\section{Article history:}

Received 23 September 2010

Received in revised form 28 April 2011

Accepted 19 July 2011

Available online 26 July 2011

\section{Keywords:}

Language

Communication

Theory of mind

Mentalizing

Brain

\begin{abstract}
A B S T R A C T
A debated issue in the relationship between language and thought is how our linguistic abilities are involved in understanding the intentions of others ('mentalizing'). The results of both theoretical and empirical work have been used to argue that linguistic, and more specifically, grammatical, abilities are crucial in representing the mental states of others. Here we contribute to this debate by investigating how damage to the language system influences the generation and understanding of intentional communicative behaviors. Four patients with pervasive language difficulties (severe global or agrammatic aphasia) engaged in an experimentally controlled non-verbal communication paradigm, which required signaling and understanding a communicative message. Despite their profound language problems they were able to engage in recipient design as well as intention recognition, showing similar indicators of mentalizing as have been observed in the neurologically healthy population. Our results show that aspects of the ability to communicate remain present even when core capacities of the language system are dysfunctional.
\end{abstract}

\section{Introduction}

How language is related to thought is a key issue in the mind sciences. A much-debated issue is how language is linked to our capacity to understand other people's intentions, thoughts, and beliefs ('mentalizing'). It has been suggested that the language system is crucially involved in representing and understanding other people's mental states (e.g. Carruthers, 2002; Newton \& de Villiers, 2007). An important theoretical reason for positing this relationship is that the recursive capacity of human language allows for representations in which one proposition is embedded inside of another (e.g. [She believes that [he is on his way to work]]; Carruthers, 2002; Pyers, 2006). Alternatively, it has been hypothesized that communicative and linguistic abilities are cognitively distinct (Bara, 2010; Levinson, 2000, 2006; Sperber \& Wilson, 1986; Tomasello, 2008; Tomasello, Carpenter, Call, Behne, \& Moll, 2005).

The empirical evidence on this issue is inconclusive (see Willems \& Varley, 2010). On the one hand, there is evidence for a necessary role of language in intention understanding. For instance, it was found that performance on a false belief task (a standard indicator of mentalizing), was diminished when participants concurrently

\footnotetext{
* Corresponding author. Tel.: +31 24 3614793; fax: +31 243610989.

E-mail address: r.willems@donders.ru.nl (R.M. Willems).
}

performed a verbal shadowing task (Newton \& de Villiers, 2007). The rationale of that study was that verbal shadowing taxes the language system and that this leads to reduced performance on mentalizing tasks. On the other hand, findings from neuroimaging studies in healthy humans (Willems et al., 2010), as well as from mentalizing performance in aphasic patients (Varley \& Siegal, 2000) seem to suggest that language and mentalizing are separable cognitive constructs. For instance, in an earlier study we found that parts of the cortical 'mentalizing network' (medial prefrontal cortex) were sensitive to a change in communicative effort, but not to a change in linguistic difficulty. Traditional language areas (e.g. left inferior frontal cortex) showed the opposite pattern of response: sensitivity to linguistic difficulty, but not to communicative effort.

These earlier results were obtained in young, healthy adults and the method of choice (fMRI) leads to correlational evidence for the involvement of a given brain area. In the current study we aimed at furthering our understanding of the relationship between mentalizing and language capacities by investigating how severe and irreversible damage to the language system impacts performance on a non-linguistic communication paradigm that forces participants to generate and interpret new intentional behaviors. Four severely aphasic participants took part in the Tacit Communication Game (TCG; de Ruiter, Noordzij, Newman-Norlund, Hagoort, \& Toni, 2007; de Ruiter et al., 2010; Newman-Norlund et al., 2009; Noordzij et al., 2009), a task in which a Sender communicates a 
A

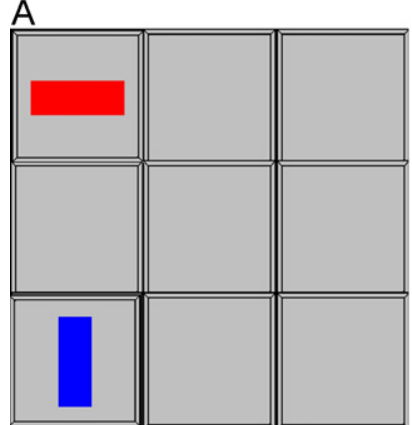

D

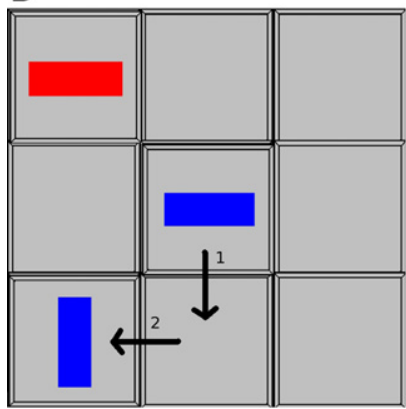

B

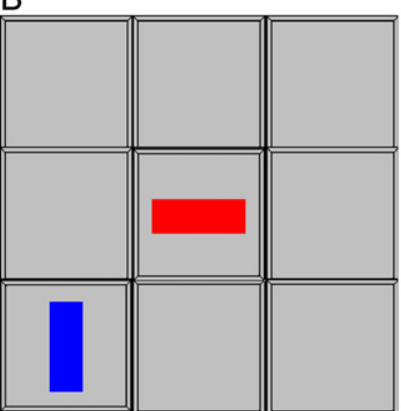

$\mathrm{E}$
C

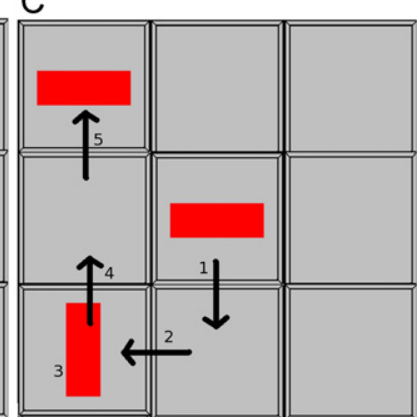

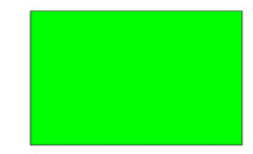

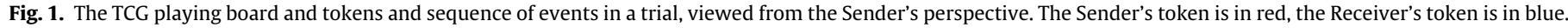

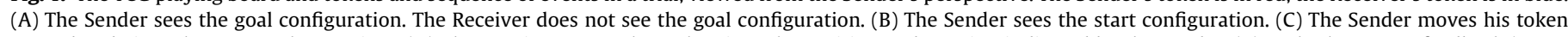

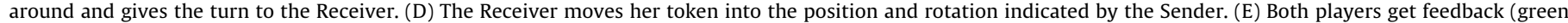

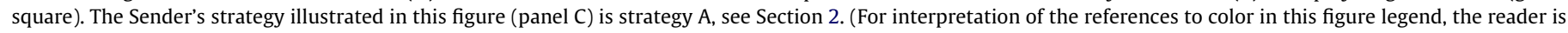
referred to the web version of the article.)

spatial configuration to another individual (Receiver) in a visuospatial setting. Importantly, the Sender knows the end-state of a trial, whereas the Receiver does not. This means that the Sender has to creatively use movement of his token across a playing board to signal where the Receiver has to move her token (Figs. 1 and 2 and Section 2). This paradigm taps into the ability to generate and understand a message signaling a communicative intention to someone else, while maintaining rigorous experimental control (see below and de Ruiter et al., 2007; Newman-Norlund et al., 2009; Noordzij et al., 2009). Specifically, depending upon the role one plays in the paradigm, the participant needs to generate a communicative message, taking knowledge of the other person into account (recipient design), or has to recognize the communicative message generated by the other (intention recognition). In the remainder of the manuscript we refer to these abilities as 'mentalizing' or 'communicative' abilities, although we are aware of the fact that recipient design and intention recognition are only part of mentalizing (see de Ruiter et al., 2007 for discussion).

If communicative message generation/understanding necessitates a functioning (syntactic) language system, individuals with severe aphasia (including agrammatism) should be unable to successfully convey the message needed to succeed on the TCG to another individual. By contrast, if communicative intention generation and understanding is cognitively distinct from language, our participants should fare well on this task.

Successful performance of the present task would constitute empirical evidence for the clinical observation that those with severe damage to the language system can retain a largely untapped communicative potential (e.g. Goodwin, 2006), a capacity that can be easily overlooked in clinical practice, particularly in the absence of methods to systematically evaluate this potential. Such evidence could provide an impetus for devising augmentative strategies for communication.
A

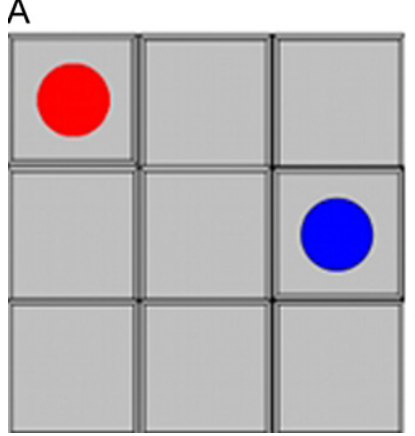

B

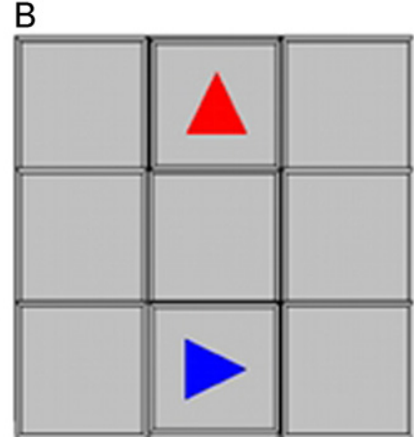

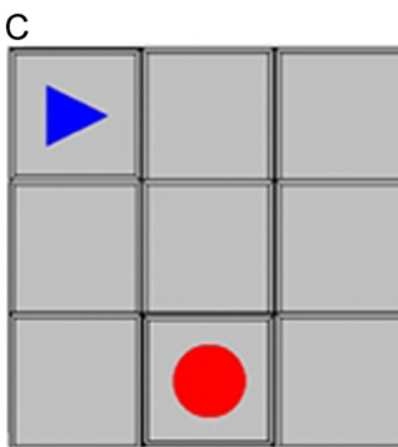

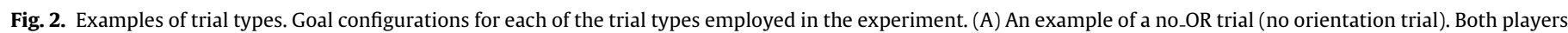

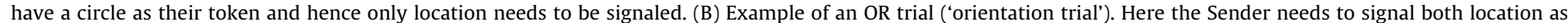

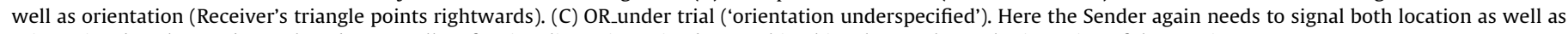
orientation, but the Sender's token does not allow for signaling orientation by matching his token to the goal orientation of the Receiver. 


\section{Materials and methods}

\subsection{Participants}

Four participants with severe aphasia were recruited (JCB, SA, PR, and ST). JCB was a 63-year-old man who was 14 years post-onset of a left hemisphere stroke in left middle cerebral artery territory. He was premorbidly right-handed and worked as a pipefitter. He displayed severe agrammatic aphasia. SA was a 64-year-old man who was 17 years post-onset of sub-dural empyema, which resulted in infarction of tissue in the left middle cerebral artery territory. He was premorbidly right-handed and had worked as a police sergeant. His brain injury resulted in severe agrammatic aphasia. PR was a 65-year-old man who was 9 years post-onset of a left middle cerebral artery stroke. He was premorbidly left-handed, but his severe aphasia following left hemisphere damage indicated that he was left hemisphere dominant for language. He had worked as a retail manager. He displayed severe agrammatic aphasia. ST was a 73-year-old man who was 9 years post-onset of a left middle cerebral artery stroke. He was premorbidly right-handed, and had worked as a company director. He had global aphasia, with profound lexical impairment in addition to grammatical processing deficits. MRI scans illustrating the extension of the lesions in three patients are provided in Supplementary Fig. S1.

Speech and written output of the agrammatic patients is confined to single words, while the global patient ST was able to produce only social speech such as 'yes' (Supplementary materials). The results of a battery of language and aphasia tests (Supplementary Table S1) confirm that the patients are markedly impaired in language production as well as in syntactic comprehension. Some lexical semantic comprehension of concrete words is retained, but this is reduced when comprehension is tested via synonym judgment tasks, which permit evaluation of understanding of abstract, low-imageability words.

\subsection{Experimental set-up}

The Tacit Communication Game (TCG) is a non-verbal communicative paradigm involving two players: a Sender and a Receiver. The goal of the task is to reach a predefined end-state by moving tokens across a $3 \times 3$ playing board (Fig. 1 ). Each player controls one token. The game is played via a computer and players can move their tokens horizontally, vertically, or rotate them by pressing keys on a keyboard.

There is an important imbalance in knowledge about the end-state which renders one player the Sender and the other the Receiver: the Sender knows what the end-state should be, whereas the Receiver does not (for ease of reference we refer to the Sender as 'he' and to the Receiver as 'she' in the remainder of the text, despite the fact that all patients were male). Both players are mutually aware that they are playing on the same workspace, with the same sensorimotor mappings, and that their movements on the workspace are visible to the other player, generating a common ground for communication. In each trial, the Sender undertakes a series of moves first and then gives the turn to the Receiver. The Receiver then decides where to move her token, and ends the trial. Feedback is given by means of a green or red square on the screen (Fig. 1).

The Sender always needs to convey the target location of the Receiver's token. Additionally, on some trials the Sender has to convey the target orientation of the Receiver's token. There are three token types: circle, rectangle and triangle (see Fig. 2). The circle has only one possible orientation, the rectangle has two possible orientations (horizontal or vertical) and the triangle has four possible orientations (pointing upwards, pointing rightwards, pointing downwards, and pointing leftwards).

By manipulating the ease of conveying orientation, different trial types are created (see Fig. 2 for examples). First, there are trials without an orientation problem (no_OR trials; Fig. 2A). These are trials in which the Receiver's token is a circle, which can have only one orientation. Therefore, the Sender has to only convey the location to the Receiver. Second, there are trials in which orientation is necessary to achieve the correct final configuration. In a subset of these trials, the geometry of the Sender's token can be mapped onto the orientation of the Receiver's token (OR trials; Fig. 2B). For instance, if both players have a rectangle as token, the Sender can indicate the Receiver's orientation by means of rotating his own rectangle. Third, there are trials in which location as well as orientation need to be signaled, but in which the Sender's token cannot be unambiguously mapped onto the orientation of the Receiver's token (Fig. 2C). For instance, if the Sender has a circle, and the Receiver a triangle, there is no way in which rotating the circle will be effective in signaling the orientation of the triangle. We call this trial type OR_under (referring to 'underspecified' to describe the token of the Sender).

Previous experiments with healthy adult participants have shown that the TCG successfully taps into communicative message generation (de Ruiter et al., 2007; Newman-Norlund et al., 2009; Noordzij et al., 2009). The most commonly observed strategy that a Sender develops is to move his token to the Receiver's location, pause, and go back to his own location. This apparently simple behavior captures a general principle of communicative message generation: pausing is an instrumentally dysfunctional behavior, and the Sender uses this property as a suggestion to the Receiver to infer a communicative intention of the Sender within the sequence of movements across the grid.

In earlier work in our laboratory, it was confirmed that this pausing strategy involves tailoring a message for a specific other. For instance, the pausing behavior changes when both players know the end-state and the Sender is required to go to the location of the Receiver because he is obliged to do so by the rules of the game (de Ruiter et al., 2007, 2010; Noordzij et al., 2009). Moreover, in another study it was shown that participants prolong their pausing behavior when they believed they were playing the game with a child as compared to with an adult, which effectively shows recipient design in the Sender, mimicking a 'motherese' way of speaking to children (Newman-Norlund et al., 2009).

\subsection{Experimental procedure}

In the first series of sessions the patients played the role of Sender and in the second series they played the role of Receiver. Sender and Receiver series were separated at least 1.5 years for three of the patients, and by 1 week for patient JCB.

Participants performed the task seated behind a computer screen. Response buttons were four arrow keys (to move token), space bar (start/stop button) and the Shift key (rotation button). The other player was one of the experimenters (RV) seated in the same room behind a different monitor. There was a large occluder between the Sender and Receiver so that Sender and Receiver could not see each other. Before the barrier was put in place, the Sender observed that the Receiver was wearing headphones and was thus unable to hear auditory signals. Each experimental session was video-recorded and behavior was examined off-line to determine if patients attempted to use communicative strategies in addition to the manipulation of his token. Recordings revealed that participants were largely silent throughout the trial, making no attempt to use vocalizations to communicate with the other participant. The Receiver was aware of the workings of the TCG, but did not know the end-state on a trial. Hence performance truly depended on the capability of the Sender to convey location and orientation to the Receiver.

In the Sender role, the patients were reminded of the end-state on each trial by means of a cardboard device showing the two tokens in their end-state position, to minimize working memory load. When the patient was in the Receiver role, the experimenter (Sender) employed the communicative strategies as outlined below.

Before the start of the experiment participants played a variable number of practice trials until they were familiar with the response buttons and moving the tokens around. In these practice trials, both Sender and Receiver could see the endstate of the game, and hence no communicative exchange was required. The task of both players was to go to their respective end-location. Participants received no training of communicative trials. After practice ended, the occluder was placed in position and participants were made aware that the Receiver was unable to see the end-state and that the goal of the game was to reach the end-state. Given the severe receptive language difficulties in all participants, instruction was visual and gestural. It was ensured that no mention of communicative strategy or of the need to communicate was made during instruction. Participants took part in eight separate sessions (four Sender, four Receiver), with 20 trials per session. Due to technical error participant ST only played three sessions as a Receiver.

\subsection{Data analysis}

Data analysis involved determining success rate and computing pausing time on Receiver's target location in comparison to the average pause times on other locations for each trial type. Earlier work has shown that the pausing time on the Receiver's target location is a good indicator of communicative message generation (de Ruiter et al., 2007, 2010; Newman-Norlund et al., 2009; Noordzij et al., 2009). Pausing times on the first and last locations were excluded since they tend to be idiosyncratically higher (de Ruiter et al., 2010), and the average pause times on the other non-target locations was computed for each trial and compared to the pause time at the target location. We used a non-parametric approach to empirically derive a distribution for inferential statistics by means of 5000 random re-orderings of the data. The actual observed $t$-statistic was compared to this generated distribution and the according $p$-value was determined as the proportion of statistics from the reference distribution which was larger than the observed $t$-statistic (Higgins, 2004).

Success rate was expressed as the percentage of correct trials for each trial type separately. A trial was correct when both Sender as well as Receiver went to the correct location and had their token turned in the correct orientation. The Receive has 8 locations she can go to (nine locations minus the location occupied by the Sender) and a variable number of orientations depending upon her token ( 0 for circle, 2 for rectangle and 4 for triangle). This means that chance level ranges between $3 \%$ ( 1 out of 32 possible combinations) and $12.5 \%$ ( 1 out of 8 possible combinations).

Another analysis involves a qualitative assessment of the strategies employed on each trial when the patients were in the Sender role. To this end we coded strategies that the Sender employed following this coding scheme (see de Ruiter et al., 2010; Galantucci, 2005 for a comparable approach):

Strategy A: Go to Receiver location, Pause/Rotate to match Receiver orientation, Go to Sender location.

Strategy B: Rotate on start location to match Receiver orientation, Go to Receiver location, Pause, Go to Sender location, rotate to Sender orientation.

Strategy C: Go to Receiver location, Pause, Go to Sender location (no rotation needed). 

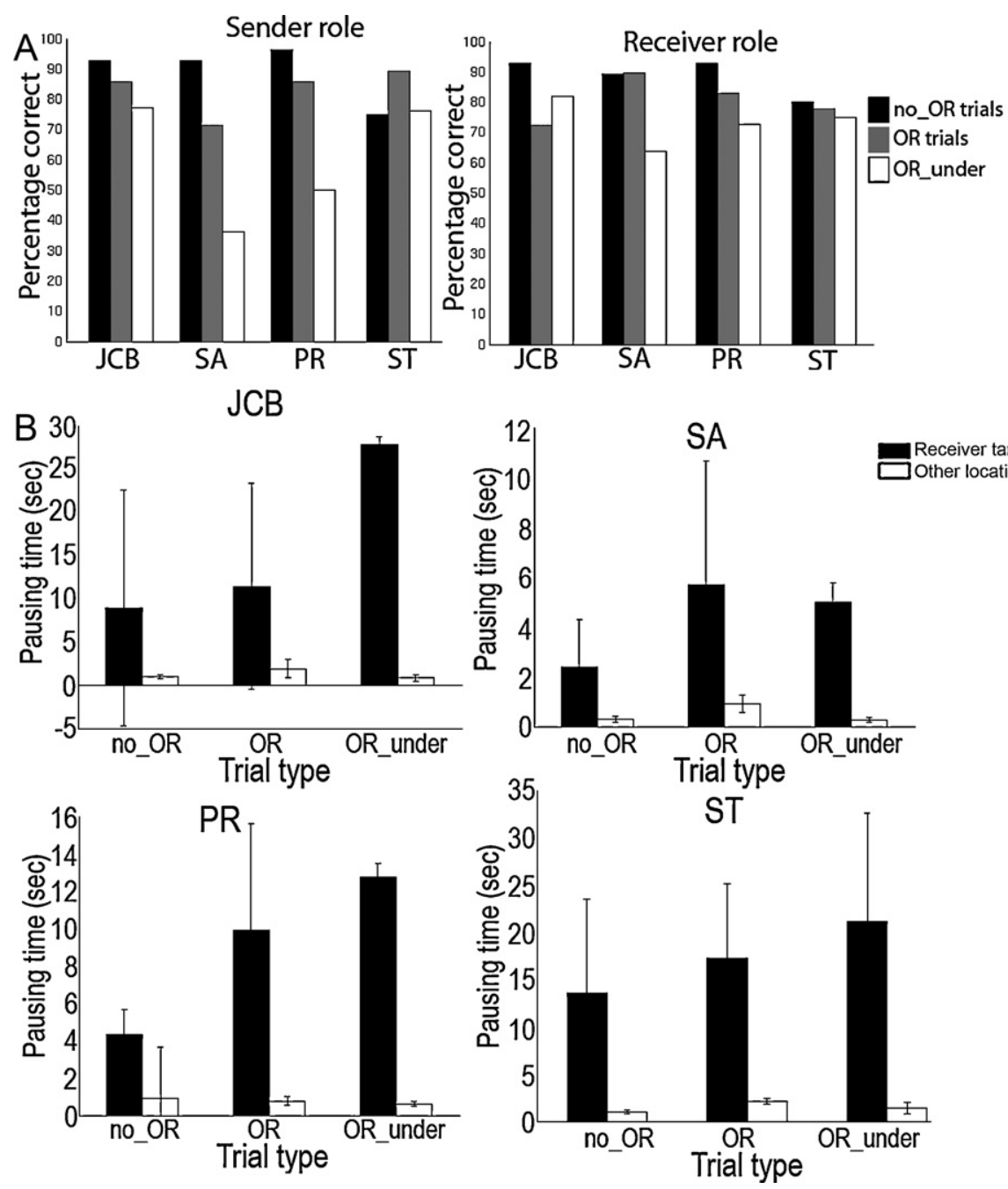

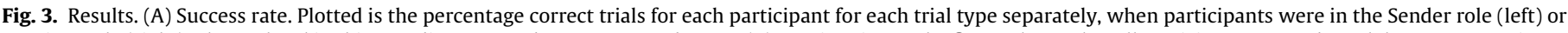

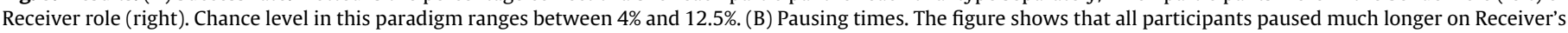
target locations as compared to other locations for all trial types. Error bars reflect standard deviation.

When in the Sender role, participants SA and ST used the strategy to first go to his own location and then go to Receiver location, and finally returning to his (Sender) location. The only difference with strategies A, B and C is that the Sender first goes to his own location, then follows the same sequence. In terms of communicative strategy these sequences are very much alike and we coded them as A2, B2 and C2.

In the communicatively most challenging trial type, the OR_under trials, two additional strategies were observed. The first is the 'rotate circle' strategy. In this strategy the Sender tries to convey the orientation of the Receiver's token by rotating his own token which is a circle. Although this is not an effective strategy (rotation of a circle is not visible on the screen), this behavior indicates that participants recognized the need to signal the Receiver's orientation. Second, participant ST employed the 'wiggle' strategy that was also occasionally observed in previous work (de Ruiter et al., 2010). In this scenario, the Sender wiggles his token between the Receiver's target location and the location next to the target location to indicate the orientation of the Receiver's token. This is an effective communicative strategy.

\section{Results}

\subsection{Success rates}

Success rate was well above chance level for all participants, both when they were in the Receiver role (JCB: $82 \%$ correct; SA: 81\%; PR: $83 \%$; ST: 79\%,), as well as when they were in the Sender role (JCB 85\%; SA: 66\%; PR: 77\%; ST: 80\%; chance level: 3-12.5\%). Success rate varied according to trial type (Fig. 3A). When they were in the Sender role, success rate was high for all players on no_OR trials (JCB: 93\%; SA: 93\%; PR: 96\%; ST: 75\%), and OR trials (JCB:
86\%; SA: 71\%; PR: 86\%; ST: 89\%). Success rate dropped considerably for the OR_under trials for two participants (SA: 36\%; PR: $50 \%$, but remained high for the other participants (JCB: 74\%; ST: 76\%). When patients were in the Receiver role, percentage correct was very high on no_OR trials (JCB: 93\%; SA: 89\%; PR: 93\%; ST 86\%), was still high on OR trials (JCB: 72\%; SA: 90\%; PR: $83 \%$; ST: 79\%), and remained high in OR_under trials (JCB: 82\%; SA: 64\%; PR: 74\%; ST: 73\%).

\subsection{Pausing times}

Pausing times were only reported when patients were in the Sender role, in order to determine if was used as communicative strategy. All Senders paused much longer on the Receiver's target location than on other locations (Fig. 3B): pausing on Receiver's target: JCB (mean 15.85, s.d. 22.18); SA (mean $4.09 \mathrm{~s}$, s.d. 5.66 s), PR (mean 8.75, s.d. 14.26); ST (mean 16.82, s.d. 17.15); pausing on other locations: JCB (mean 1.48, s.d. 1.24); SA (mean 0.29s, s.d. 0.29); PR (mean 0.80, s.d. 1.82); ST (mean 1.56, s.d. 1.22), which is a communicative strategy also observed in the neurologically healthy population (see Section 2 for details). Statistical analysis showed these differences to be stable and statistically significant for each participant and each trial type (JCB: no_OR: $t(27)=2.61, r=0.23, p<0.001$; OR: $t(27)=3.15, r=0.29$, $p<0.001$; OR_under: $t(24)=3.96, r=0.46, p<0.001$; SA: no_OR: 
Table 1

Strategies used in the different trial types. The table shows the number of trials on which a given strategy was employed, separately calculated for each trial type.

\begin{tabular}{|c|c|c|c|c|c|c|c|c|c|c|c|c|c|c|c|c|}
\hline & \multicolumn{8}{|l|}{$\mathrm{JCB}$} & \multicolumn{8}{|l|}{ SA } \\
\hline & \multicolumn{8}{|c|}{ Strategy used (number of trials) } & \multirow[b]{2}{*}{$n$ (trials) } & \multirow[b]{2}{*}{ A } & \multirow[b]{2}{*}{$\mathrm{B}$} & \multirow[b]{2}{*}{ C } & \multirow[b]{2}{*}{$\mathrm{A} 2$} & \multirow[b]{2}{*}{ B2 } & \multirow[b]{2}{*}{$\mathrm{C} 2$} & \multirow[b]{2}{*}{ Unclass } \\
\hline & $n$ (trials) & A & B & C & A2 & B2 & $\mathrm{C} 2$ & Unclass & & & & & & & & \\
\hline no_OR & 28 & 1 & 0 & 27 & 0 & 0 & 0 & 0 & 28 & 1 & 0 & 22 & 0 & 0 & 3 & 2 \\
\hline OR & 28 & 14 & 0 & 11 & 1 & 0 & 0 & 2 & 28 & 5 & 5 & 6 & 10 & 1 & 0 & 1 \\
\hline OR_under & 22 & 1 & 0 & 19 & 0 & 0 & 0 & 2 & 22 & 6 & 0 & 16 & 0 & 0 & 0 & 0 \\
\hline \multirow[t]{4}{*}{ Trials (total) } & 78 & 16 & 0 & 57 & 1 & 0 & 0 & 4 & 78 & 12 & 5 & 44 & 10 & 1 & 3 & 3 \\
\hline & \multicolumn{8}{|l|}{ PR } & \multicolumn{8}{|l|}{ ST } \\
\hline & \multicolumn{8}{|c|}{ Strategy used (number of trials) } & \multicolumn{8}{|c|}{ Strategy used (number of trials) } \\
\hline & $n$ (trials) & A & $\mathrm{B}$ & $\mathrm{C}$ & $\mathrm{A} 2$ & B2 & $\mathrm{C} 2$ & Unclass & $n$ (trials) & A & B & $\mathrm{C}$ & A2 & B2 & $\mathrm{C} 2$ & Unclass \\
\hline no_OR & 28 & 4 & 0 & 23 & 0 & 0 & 0 & 1 & 28 & 0 & 0 & 9 & 2 & 0 & 15 & 2 \\
\hline OR & 28 & 10 & 6 & 9 & 0 & 0 & 0 & 3 & 28 & 3 & 5 & 0 & 19 & 0 & 0 & 1 \\
\hline OR_under & 22 & 3 & 0 & 19 & 0 & 0 & 0 & 0 & 22 & 3 & 0 & 2 & 14 & 1 & 0 & 2 \\
\hline Trials (total) & 78 & 17 & 6 & 51 & 0 & 0 & 0 & 4 & 78 & 6 & 5 & 11 & 35 & 1 & 15 & 5 \\
\hline
\end{tabular}

$t(27)=3.87, r=0.36, p<0.001 ;$ OR: $t(27)=2.87, r=0.23, p<0.001$; OR_under: $t(21)=4.22, r=0.46, p<0.001$; PR: no_OR: $t(26)=4.15$, $r=0.40, p<0.001$; OR: $t(27)=4.74, r=0.45, p<0.001$; OR_under: $t(21)=2.45, r=0.22, p<0.001 ;$ ST: no_OR: $t(27)=3.90, r=0.36$, $p<0.001$; OR: $t(27)=4.88, r=0.47, p<0.001$; OR_under: $t(20)=4.51$, $r=0.50, p<0.001)$.

Comparison of pausing times on the Receiver's target for the different trial types showed that there was a trend for longer pausing times for OR as compared to no_OR trials (SA: $t(54)=1.54$, $r=0.05, p=0.08$; PR: $t(53)=2.96, r=0.14, p=0.002$; ST: $t(54)=1.01$, $r=0.02, p=0.33)$, except for in patient JCB $(t(54)=1.34, r=0.03$, $p=0.22$ ). Pausing in the OR_under trials was significantly longer than in the no_OR trials, although there was only a trend in participant ST (JCB: $t(48)=3.19, r=0.18, p=0.002$; SA: $t(48)=1.95, r=0.07$, $p=0.05$; PR: $t(47)=1.93, r=0.07, p=0.013$; ST: $t(47)=1.80, r=0.07$, $p=0.07)$. Pausing times did not differ between OR and OR_under trials (JCB: $t(48)=2.09, r=0.08, p=0.05$; SA: $t(48)<1, r<0.01, p>0.5$; PR: $t(48)<1, r<0.01, p>0.5$; ST: $t(47)<1, r=0.02, p=0.33)$.

\subsection{Communicative strategies}

Participants employed strategies with a clear communicative component in the large majority of trials (see Section 2 and Table 1 ). Only in a minor portion of trials could the communicative strategy not be established (JCB: 4/78; SA: 3/78 trials; PR: 4/78; ST: 5/78;).

In no_OR trials, in which no rotations are needed to signal orientation, the large majority of trials did not involve rotations $(27 / 28$, $25 / 28,23 / 28$, and $24 / 28$, trials for JCB, SA, PR, and ST, respectively, as compared to $1 / 28,1 / 28,4 / 28$, and $2 / 28$ trials involving rotations; Table 1). By contrast, strategies involving rotations were employed in the OR trials $(15 / 28,21 / 28,16 / 28$, and $27 / 28$, as compared to $14 / 28,6 / 28,9 / 28$, and $0 / 28$ trials not involving rotations; Table 1).

In the OR_under trials (the most difficult trials), two participants employed the C strategy (go to Receiver's target location, Pause, go the Sender's target location) in the large majority of trials (16/22 and 19/22 for SA and PR, respectively). This is not an efficient strategy for signaling the orientation of the Receiver's token and as we saw above, success rate was correspondingly low (36\% and $50 \%$ ). This was not the case for participants ST and JCB. They also employed the $\mathrm{C}$ and $\mathrm{C} 2$ strategies, but additionally used the 'rotate_circle' (ST) and the more effective 'wiggle' strategy (ST and JCB) in several OR_under trials and performance was accordingly higher (76\% and $74 \%$ correct). We will elaborate on this finding in the discussion section.

\section{Discussion}

We tested whether the language system is obligatorily involved when we design or understand a communicative act (Carruthers, 2002; Newton \& de Villiers, 2007). Here we provide evidence against this conjecture. Patients with very severe and irrevocable damage to components of language hypothesized to be crucial for mentalizing (most notably syntax) were able to design (recipient design) as well as interpret (intention recognition) novel communicative actions in a visuo-spatial communication paradigm in similar ways as done by non-aphasic samples (de Ruiter et al., 2007, 2010; Newman-Norlund et al., 2009; Noordzij et al., 2009). This finding is evidence for the thesis that subsets of communicative and linguistic abilities rely on distinct neural and cognitive substrates (e.g. Bara, 2010; Levinson, 2006; Tomasello, 2008; Tomasello et al., 2005). More specifically, it shows that recipient design and intention recognition are neurally distinct from other language capacities.

We employed a visuo-spatial communication paradigm dubbed the 'Tacit Communication Game' (TCG). In this paradigm one participant (the Sender) has to communicate location and orientation of the other player's (the Receiver) token by navigating a token on a grid. There is an imbalance in knowledge between the two players: the Sender knows where the Receiver has to go to, but the Receiver does not know where she has to go. An important reason to posit that the patients were generating a communicative act is the finding that pausing times were much longer on the location that had to be communicated to the Receiver. Also, Senders rather effectively communicated the orientation of the Receiver's token by using several strategies that have been observed before in the neurologically healthy population (de Ruiter et al., 2007, 2010; Newman-Norlund et al., 2009; Noordzij et al., 2009).

It could be argued that the behavior we observed in the TCG reflects solving a logical puzzle, without any necessary link to the generation of communicative acts. However, several observations from the present as well as other studies do not fit with this interpretation. First, previous work has shown that the communicative actions evoked in the TCG are influenced by the perceived mental state of the receiver. For instance, adult participants adjust their communicative behavior depending upon whether they belief to be playing the game with a child or with another adult (NewmanNorlund et al., 2009). Second, the Sender stops pausing on the Receiver's location when he is told that the Receiver already knows where to position her token (de Ruiter et al., 2007, 2010; Noordzij et al., 2009). Third, in the present study the Sender's behavior was tailored to the Receiver, taking her lack of knowledge into 
account. Each patient exploited the pausing behavior in a similar manner as observed in healthy young volunteers, taking account of its dysfunctional characteristics (with respect to the sensorimotor constraints of the task) to tag communicative elements of their actions. Also all patients recognized the need to signal the Receiver's orientation and tried to communicate this orientation. Importantly this was only done in trial types in which there was a need for the Receiver to actually alter the orientation of her token. For instance, Senders did not rotate their token when the Receiver's token was a circle (which can have only one orientation). Taken together, these observations indicate that the TCG effectively tapped into communicative message generation.

Our present finding is in line with a previous neuroimaging study in which we found that distinct neural substrates are influenced by generation of a communicative act versus general semantic language processing (Willems et al., 2010). In that study it was found that in young healthy volunteers the dorso-medial prefrontal cortex, an area which is part of the 'mentalizing' network (Frith \& Frith, 2006), was sensitive to whether a message was generated for a specific other or not. By contrast, part of the cortical language network (left inferior frontal cortex) was influenced by how difficult it was to construct a message in linguistic terms, but was insensitive to a difference in communicative message generation. It should be noted that the locus of activation sensitive to the communicative manipulation in our previous study (dorso-medial prefrontal cortex) seems to be intact in the patients that took part in the present study (Supplementary Fig. S1). Future work should be aimed at investigating how these independent neural systems interact in the most prominent case of everyday communication, namely when we use language to communicate.

It should be noted that our data are pertinent to the role of language in the mature or adult cognitive system and cannot be taken to imply that language plays no role in the development of mentalizing capacities. All patients in the present experiment were fluent users of language prior to insult which occurred only in a later phase of life, and far beyond the time period in which language and mentalizing capacities emerge.

The TCG has clinical potential both in evaluating the integrity of non-linguistic message generation in brain-injured individuals and in developing new intervention strategies. First, such methods provide a way of objectively recording and quantifying communicative ability. While in clinical contexts, some individuals with aphasia have been observed as using alternative communicative resources such as drawing and gesture to convey their message, this is not the case with all severely aphasic individuals. The reporting of interventions to develop drawing abilities and also the difficulties some patients have in generating communicative drawing is an indication that residual communicative capacity cannot be assumed to be present in individuals with severe aphasia (Sacchett \& Black, 2011). Second, methods such as the TCG might be useful in communicative rehabilitation through facilitating awareness of what a recipient may or may not know, and in stimulating an individual to seek alternative communicative strategies in the face of loss of access to linguistic resources. In this way, the TCG or similar methods might enhance the effectiveness of interventions such as drawing therapy. If used as a precursor to the communicative therapy, the method can highlight the need to consider recipient design in formulating a communicative message.

\section{Acknowledgements}

Supported by European Union Joint-Action Science and Technology Project (IST-FP6-003747), the Netherlands Organisation for Scientific Research (NWO-Rubicon 446-08-008 to RW and NWOVICI 016-095-613 to IT), the Niels Stensen Foundation and the UK ESRC (RES-051-27-0189). We are greatly indebted to JCB, PR, SA and ST and their families. We thank three anonymous reviewers for their insightful comments on an earlier version of the paper. Marek Tyc is acknowledged for assistance with computer hardware.

\section{Appendix A. Supplementary data}

Supplementary data associated with this article can be found, in the online version, at doi:10.1016/j.neuropsychologia.2011.07.023.

\section{References}

Bara, B. G. (2010). Cognitive pragmatics. Cambridge, MA: MIT Press.

Carruthers, P. (2002). The cognitive functions of language. Behavioral and Brain Sciences, 25(6), 657-726

de Ruiter, J. P., Noordzij, M. L., Newman-Norlund, S., Hagoort, P., \& Toni, I. (2007). On the origin of intentions. In Y. Rossetti, P. Haggard, \& M. Kawato (Eds.), Sensorimotor foundations of higher cognition (pp. 593-610). Oxford: Oxford University Press.

de Ruiter, J. P., Noordzij, M. L., Newman-Norlund, S., Newman-Norlund, R., Hagoort, P., Levinson, S. C., et al. (2010). Exploring the cognitive infrastructure of communication. Interaction Studies, 11, 51-77.

Frith, C. D., \& Frith, U. (2006). The neural basis of mentalizing. Neuron, 50(4), 531-534.

Galantucci, B. (2005). An experimental study of the emergence of human communication systems. Cognitive Science, 29(5), 737-767.

Goodwin, C. (2006). Human sociality as mutual orientation in a rich interactive environment: Multimodal utterances and pointing in aphasia. In N. J. Enfield, \& S. C. Levinson (Eds.), Roots of human sociality (pp. 97-125). New York: Berg.

Higgins, J. J. (2004). Introduction to modern nonparametric statistics. Pacific Grove, CA: Brooks Cole.

Levinson, S. C. (2000). Presumptive meanings. Cambridge, MA: MIT Press.

Levinson, S. C. (2006). On the human interactional engine. In N. J. Enfield, \& S. C. Levinson (Eds.), Roots of human sociality. New York: Berg.

Newman-Norlund, S. E., Noordzij, M. L., Newman-Norlund, R. D., Volman, I. A., Ruiter, J. P., Hagoort, P., et al. (2009). Recipient design in tacit communication. Cognition, 111(1), 46-54

Newton, A. M., \& de Villiers, J. G. (2007). Thinking while talking: Adults fail nonverbal false-belief reasoning. Psychological Science, 18(7), 574-579.

Noordzij, M. L., Newman-Norlund, S. E., de Ruiter, J. P., Hagoort, P., Levinson, S. C., \& Toni, I. (2009). Brain mechanisms underlying human communication. Frontiers in Human Neuroscience, 3, 14.

Pyers, J. E. (2006). Constructing the social mind: Language and false-belief understanding. In N. J. Enfield, \& S. C. Levinson (Eds.), Roots of human sociality. New York: Berg.

Sacchett, C., \& Black, M. (2011). Drawing as a window to event conceptualisation: Evidence from two people with aphasia. Aphasiology, 25, 3-26.

Sperber, D., \& Wilson, D. (1986). Relevance; communication and cognition. Oxford, UK: Basil Blackwell.

Tomasello, M. (2008). Origins of human communication. Cambridge, MA: MIT Press.

Tomasello, M., Carpenter, M., Call, J., Behne, T., \& Moll, H. (2005). Understanding and sharing intentions: The origins of cultural cognition. Behavioral and Brain Sciences, 28(5), 675-691.

Varley, R., \& Siegal, M. (2000). Evidence for cognition without grammar from causal reasoning and 'theory of mind' in an agrammatic aphasic patient. Current Biology, 10(12), 723-726.

Willems, R. M., de Boer, M., de Ruiter, J. P., Noordzij, M. L., Hagoort, P., \& Toni, I. (2010). A cerebral dissociation between linguistic and communicative abilities in humans. Psychological Science, 21(1), 8-14.

Willems, R. M., \& Varley, R. (2010). Neural insights into the relation between language and communication. Frontiers in Human Neuroscience, 4, 203. 